\title{
BMJ Open Information resources to aid parental decision-making on when to seek medical care for their acutely sick child: a narrative systematic review
}

\author{
Sarah Neill, ${ }^{1}$ Damian Roland, ${ }^{2,3}$ Caroline HD Jones, ${ }^{4}$ Matthew Thompson, ${ }^{5}$ \\ Monica Lakhanpaul, ${ }^{6}$ on behalf of the ASK SNIFF study group
}

To cite: Neill S, Roland D, Jones CHD, et al. Information resources to aid parental decision-making on when to seek medical care for their acutely sick child: a narrative systematic review. BMJ Open 2015;5:e008280

doi:10.1136/bmjopen-2015008280

\section{- Prepublication history} and additional material is available. To view please visit the journal (http://dx.doi.org/ 10.1136/bmjopen-2015008280).

Received 25 March 2015 Revised 1 October 2015 Accepted 17 October 2015

CrossMark

For numbered affiliations see end of article.

\section{Correspondence to}

Professor Monica

Lakhanpaul;

m.lakhanpaul@ucl.ac.uk

\section{ABSTRACT}

Objective: To identify the effectiveness of information resources to help parents decide when to seek medical care for an acutely sick child under 5 years of age, including the identification of factors influencing effectiveness, by systematically reviewing the literature.

Methods: 5 databases and 5 websites were systematically searched using a combination of terms on children, parents, education, acute childhood illness. A narrative approach, assessing quality via the Mixed Methods Appraisal Tool, was used due to noncomparable research designs.

Results: 22 studies met the inclusion criteria: 9 randomised control trials, 8 non-randomised intervention studies, 2 qualitative descriptive studies, 2 qualitative studies and 1 mixed method study. Consultation frequency (15 studies), knowledge (9 studies), anxiety/reassurance (7 studies), confidence (4 studies) satisfaction (4 studies) and antibiotic prescription (4 studies) were used as measures of effectiveness. Quality of the studies was variable but themes supported information needing to be relevant and comprehensive to enable parents to manage an episode of minor illness Interventions addressing a range of symptoms along with assessment and management of childhood illness, appeared to have the greatest impact on the reported measures. The majority of interventions had limited impact on consultation frequencies, No conclusive evidence can be drawn from studies measuring other outcomes.

Conclusions: Findings confirm that information needs to be relevant and comprehensive to enable parents to manage an episode of minor illness. Incomplete information leaves parents still needing to seek help and irrelevant information appears to reduce parents' trust in the intervention. Interventions are more likely to be effective if they are also delivered in non-stressful environments such as the home and are coproduced with parents.

\section{BACKGROUND}

Acute illness is a universal experience for children and families and represents the most common type of illness in childhood,
Strengths and limitations of this study

- This is the first review of the outcome of information resources which aid parental decisionmaking utilising systematic search and quality assessment criteria

- The strengths of this review lies in its inclusiveness. Using an integrative narrative approach enabled us to identify influences on effectiveness across a wider range of studies and topics than would have been possible with a single study type or topic focused review.

- The findings are limited by the quality of the studies and not being able to control for the impact of different healthcare delivery systems.

particularly in $0-5$-year-olds. Acute illness includes short-term illnesses, predominantly infections such as coughs, colds, diarrhoea, vomiting and ear infections. Home management is often supported by consultations in primary care, where children under 5 years old constitute $40 \%$ of general practitioner (GP) workload, ${ }^{1}$ with most consultations for acute illness. ${ }^{2}{ }^{3}$ Under 1-year olds are seen more often than all other age groups other than the over $75 \mathrm{~s}^{2}$ and urgent care and emergency department service use by young children appears to be rising. ${ }^{4-6}$

Parents' anxiety about acute childhood illness leads them to seek information to help them decide whether or not to seek help from a healthcare professional. ${ }^{7-11}$ A wide range of information is available for families, such as written leaflets or via websites much of which is either unknown to parents ${ }^{5}$ or does not seem to be making any impact on service use when children are acutely sick at home. ${ }^{11-14}$ The increase in consultation rates for non-urgent care ${ }^{4-6}$ suggests more effective information sources are needed. 
We aimed to systematically review the literature to identify the effectiveness of information resources to help parents decide when to seek medical care for an acutely sick child under 5 years of age, including the identification of factors influencing effectiveness.

Our research questions were:

- What measures of effectiveness have been used to evaluate such interventions?

- How effective are existing interventions in helping parents know when to seek help for an acutely sick child at home?

- What factors influence effectiveness of information provision to help parents know when to seek help for an acutely sick child at home?

\section{METHODS}

\section{Search strategy}

We systematically searched five electronic databases (MEDLINE, CINAHL, PsycNET, ASSIA Web of Knowledge) and five websites (Centre for Review and Dissemination York, National Institute for Health and Care Excellence, Health Technology Assessment programme, NHS Evidence and the Cochrane Library) using a combination of terms on children, parents/ carers, education, acute childhood illness (see online supplementary appendix 1). We scanned reference lists of key articles, and attempted to contact authors when further information was required to determine eligibility and inform quality assessment.

\section{Selection criteria}

Studies which met all the following criteria were included:

1. Studies which included children from 0 to 14 years with research participants being their parents or caregivers. Initial pilot searches aimed solely at children under 5 years yielded minimal results.

2. An educational intervention on acute childhood illness was provided to parents/caregivers in any form (written, visual, verbal or electronic) designed to help with decision-making about whether or not to seek medical help.

3. The study was conducted in primary care, emergency departments, ambulatory settings or in the home, in high income countries as defined by Organisation for Economic Co-operation and Development (OECD). We included all study types.

Studies were excluded if they focused on chronically ill children, hospital inpatient settings or educational interventions designed for health professionals. We limited our search to papers published in the English language, between January 1990 and June 2014 (inclusive). The decision to search from 1990 was taken pragmatically as health services have evolved considerably since the latter half of the 20th century. We did not exclude studies on the basis of quality alone but have noted the quality of studies when discussing their impact. To have excluded low quality studies would have reduced the comprehensiveness of the review, especially given the likely heterogeneity of study design.

The titles and abstracts of studies identified in the search were retrieved and assessed by one reviewer who excluded those that were clearly not relevant. The full text of remaining studies was assessed for inclusion by two reviewers; discrepancies were resolved by discussion between all authors. Reasons for exclusion were recorded (see online supplementary appendix 2 ).

\section{Data extraction and quality assessment}

Data from included studies were extracted by one reviewer and checked by a second reviewer. All studies which met the inclusion criteria were included regardless of quality, which was assessed independently by two other reviewers using the Mixed Methods Appraisal Tool (MMAT) ${ }^{15}$ This gives a rating between zero stars (lowest quality) and 4 stars (****, highest quality).

\section{Evidence synthesis: synthesising qualitative and quantitative research}

Narrative review was used to summarise and explain findings across studies. ${ }^{1617}$ Meta-analysis was inappropriate due to non-comparable research designs.

\section{RESULTS}

The search identified 7863 studies, of which 22 were included (figure 1). Table 1 shows the characteristics of included studies of which there were nine randomised controlled trials, eight non-randomised intervention studies, two qualitative descriptive studies, two qualitative studies and one mixed method study. Thirteen were conducted in the USA, six in the UK, two in Canada and one in Denmark. Parents/caregivers of children aged $0-14$ years were included across all studies, with 12 studies limiting inclusion to parents of children under the age of 6 years. Studies were conducted in primary care (9), emergency department/hospital (7), child health clinics (3) and children's health centres (3).

Interventions involved written information in all but one study, which used video alone. ${ }^{18}$ Written information was augmented by video/slide presentations, ${ }^{19-23}$ home visits, ${ }^{12}{ }^{24}$ reinforcement within consultations ${ }^{19} 2325-28$ or was part of a structured educational programme. ${ }^{29-31}$ Three separate studies reported on the same 'Baby Check' intervention in different settings/ populations. ${ }^{24} 3233$

Quality of included studies is summarised in table 1, and detailed in online supplementary appendix 3. Only two studies were given the highest quality score, with many being given low scores, often due to insufficient reporting of methods.

\section{Measures of effectiveness}

The most frequently used measures of effectiveness were: consultation frequency (15 studies), parent knowledge (9 
Figure 1 Flow of information through the phases of the selection process (using PRISMA Flow Diagram structure (Moher et al., 2009)). Refer to appendix 2 for reasons for exclusion.

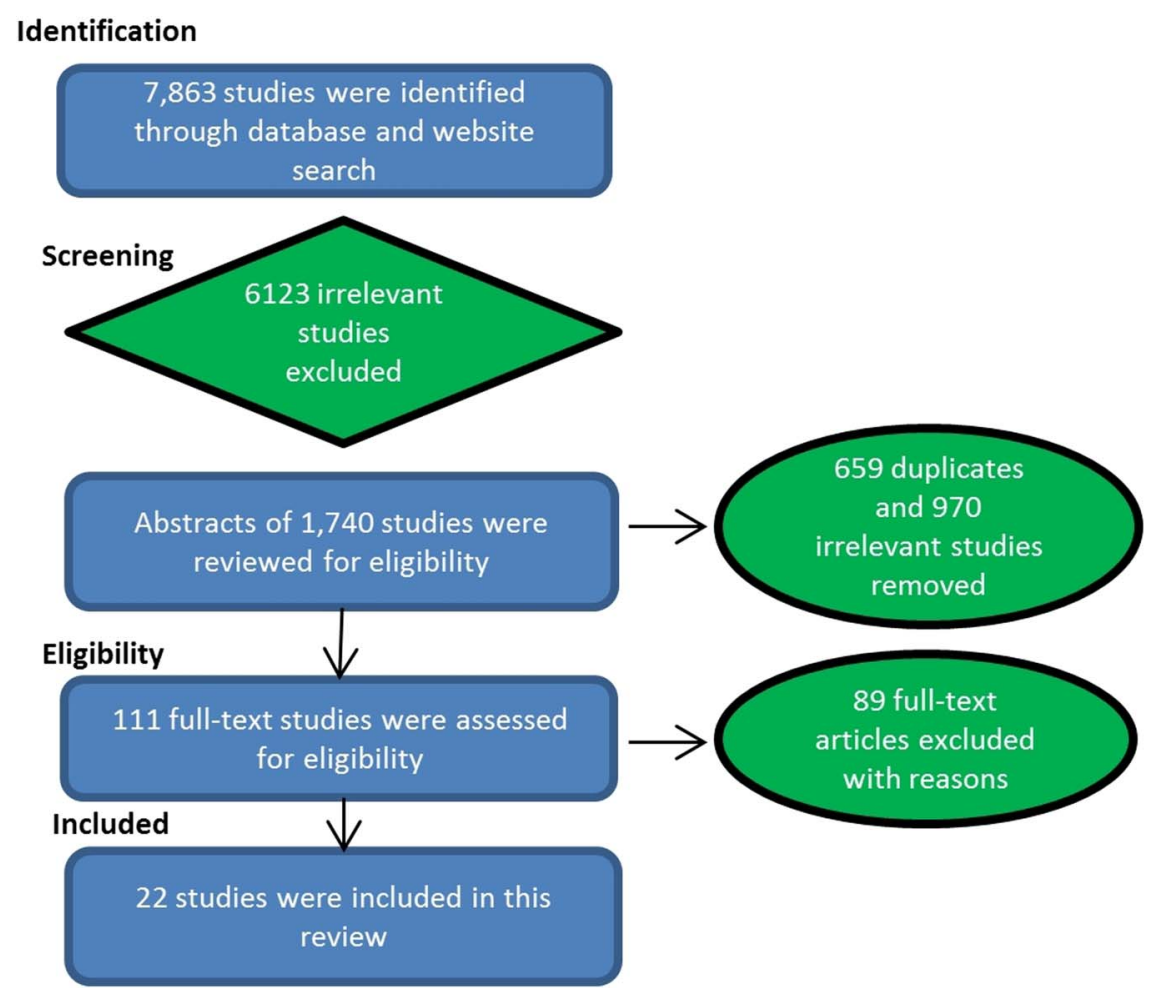

studies), parent anxiety/reassurance (7 studies), parent satisfaction (4 studies), parent confidence and clinician antibiotic prescribing (both 4 studies).

\section{Consultation frequency}

Six of the 15 studies which measured this outcome showed a significant reduction in either actual consultation rates or intention to consult in the future (see table 2). Three of these studies evaluated effects on consultation rates over a longer (1-3-year) period postintervention and found persistence of effect. ${ }^{29} 3435$ (2 low and 1 high quality). One study (low quality) showed a reduction in home visits but with an increase in out-of-hours visits. ${ }^{35}$ The eight remaining studies on consultant frequency showed no difference on consultation rates with the specified intervention.

\section{Knowledge}

Nine studies assessed the effect of interventions on parental knowledge of childhood illnesses including fever, upper respiratory infections, febrile convulsion and otitis media (see table 3). Most interventions used multiple methods to provide information, such as written materials supported by verbal explanations (one high-quality study). ${ }^{12} 192223272836$ Timing of outcome measurement ranged from immediately to 32 months later. Eight studies (one high quality) found a significant increase in parental knowledge after interven-

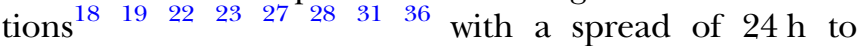
12 months for postintervention reassessment. One (high quality) study showed reduction in knowledge at 7 months. $^{12}$

\section{Anxiety/reassurance}

Of the seven randomised controlled studies that reported this outcome (table 4), only one reported significantly reduced concern compared with control group following intervention ${ }^{26}$ ( $2^{*}$ quality rating). Using Baby Check to score their baby's illness reassured $41 \%(14 / 34)^{32}$ and $46 \%{ }^{24}$ of parents, respectively. In Herman and Jackson's ${ }^{29}$ (high-quality) study the percentage of parents reporting that they were 'very worried' when their child was sick reduced by one-third.

\section{Satisfaction}

Four studies assessed the effects of interventions on parent's satisfaction with their communication with health professionals, ${ }^{19}{ }^{25}$ and with the educational information received. ${ }^{27} 37$ Two studies reported nonsignificantly increased satisfaction in control and interventions groups ${ }^{1925}$ (one high quality), while another reported significantly increased satisfaction for both intervention groups compared to controls ${ }^{27}$ ( $2 *$ quality). The fourth study suggested a web-based self-triage tool would be well received by parents ${ }^{37}$ (low quality).

\section{Confidence}

Two of four studies ${ }^{12} 19$ (one high quality) measuring the effect of interventions on parents' confidence in managing childhood illness at home did not show an increase in levels of confidence. However, Thornton et $a l \mathrm{~s}^{24}$ (high quality) field trials of 'Baby Check' found parents' confidence in the tool itself increased over time, while Kai's ${ }^{32}$ (2* quality) qualitative exploration 


\begin{tabular}{|c|c|c|c|c|c|c|c|}
\hline Author(s)/Date & Setting & Aim & Design & Sample & Intervention & Main outcomes & $\begin{array}{l}\text { Quality } \\
\text { assessment }^{*}\end{array}$ \\
\hline \multicolumn{8}{|l|}{ Qualitative studies } \\
\hline Kai $1994^{32}$ & $\begin{array}{l}\text { Health visitor and } \\
\text { general } \\
\text { practitioner baby } \\
\text { clinics (UK) }\end{array}$ & $\begin{array}{l}\text { To explore disadvantaged } \\
\text { parents' perceptions and } \\
\text { use of the Baby Check } \\
\text { booklet }\end{array}$ & $\begin{array}{l}\text { Qualitative } \\
\text { interview and } \\
\text { records of } \\
\text { consultations }\end{array}$ & $\begin{array}{l}\text { Parents of } 34 \text { babies } \\
<6 \text { months attending } \\
\text { weekly baby clinic in } \\
\text { GP in disadvantaged } \\
\text { area }\end{array}$ & $\begin{array}{l}\text { Parents were given a } \\
\text { copy of Baby Check. } \\
\text { Unstructured 30- } \\
90 \text {-min interviews with } \\
\text { parents until baby was } \\
6 \text { months }\end{array}$ & $\begin{array}{l}\text { Perceptions, use of the } \\
\text { booklet and } \\
\text { consultations for illness } \\
\text { among disadvantage } \\
\text { parents }\end{array}$ & $\star \star$ \\
\hline Krantz $2001^{38}$ & $\begin{array}{l}\text { Parent Resource } \\
\text { Centre. } \\
\text { Children's } \\
\text { Hospital Ontario } \\
\text { (Canada) }\end{array}$ & $\begin{array}{l}\text { To describe the } \\
\text { development of, and pilot, } \\
\text { a fever anticipatory } \\
\text { guidance tool for parents }\end{array}$ & $\begin{array}{l}\text { Qualitative } \\
\text { interview }\end{array}$ & $\begin{array}{l}15 \text { first-time parents } \\
\text { with children aged } \\
2 \text { months to } 4 \text { years } \\
\text { from inner city } \\
\text { Parent Resource } \\
\text { Centre }\end{array}$ & $\begin{array}{l}\text { The Fever Anticipatory } \\
\text { Guidance Tool }\end{array}$ & $\begin{array}{l}\text { Views on, and use of, } \\
\text { the booklet }\end{array}$ & * \\
\hline \multicolumn{8}{|c|}{ Randomised controlled trials } \\
\hline $\begin{array}{l}\text { Baker et al } \\
2009^{18}\end{array}$ & ED (USA) & $\begin{array}{l}\text { Effect of a brief educational } \\
\text { video during ED visit for } \\
\text { minor febrile illnesses }\end{array}$ & $\mathrm{RCT}$ & $\begin{array}{l}280 \text { parents of } \\
\text { children aged } \\
3 \text { months to } 3 \text { years } \\
\text { presenting to with } \\
\text { febrile illness }\end{array}$ & $\begin{array}{l}\text { Intervention: } 11 \text {-min } \\
\text { video on home } \\
\text { management of fever. } \\
\text { Control: 8-min video on } \\
\text { home and automobile } \\
\text { safety }\end{array}$ & $\begin{array}{l}\text { Knowledge, attitudes, } \\
\text { and return ED visits for } \\
\text { minor febrile illnesses } \\
\text { within } 2 \text { years }\end{array}$ & $\star \star \star *$ \\
\hline $\begin{array}{l}\text { Broome et al } \\
2003^{19}\end{array}$ & $\begin{array}{l}6 \text { clinics in } 6 \\
\text { states (USA) }\end{array}$ & $\begin{array}{l}\text { Effect of a structured } \\
\text { education programme on } \\
\text { parents'/grandparents' } \\
\text { knowledge, confidence, } \\
\text { and satisfaction in } \\
\text { assessing and managing a } \\
\text { child's fever }\end{array}$ & $\mathrm{RCT}$ & $\begin{array}{l}216 \text { children from } \\
3 / 12 \text { to } 6 \text { years of } \\
\text { age and their } \\
\text { parents/ } \\
\text { grandparents. } \\
183 \text { followed up at } \\
3 \text { months and } 145 \text { at } \\
6 \text { months }\end{array}$ & $\begin{array}{l}\text { Intervention 1: video } \\
\text { and brochure on } \\
\text { childhood fever in } \\
\text { clinic; } \\
\text { Intervention 2: } \\
\text { brochure and video in } \\
\text { clinic, plus health } \\
\text { professional reinforced } \\
\text { content and answered } \\
\text { parents' questions } \\
\text { during consultation; } \\
\text { Control: usual' care }\end{array}$ & $\begin{array}{l}\text { Knowledge, confidence, } \\
\text { and satisfaction in } \\
\text { assessing and } \\
\text { managing child's fever } \\
\text { at } 48 \mathrm{~h}, 1,3 \text {, and } \\
6 \text { months } \\
\text { postintervention }\end{array}$ & * \\
\hline $\begin{array}{l}\text { Chande et al } \\
1996^{20}\end{array}$ & $\begin{array}{l}\text { Urban paediatric } \\
\text { ED (USA) }\end{array}$ & $\begin{array}{l}\text { Effect of educational } \\
\text { intervention on common } \\
\text { childhood illness on ED } \\
\text { visits }\end{array}$ & $\mathrm{RCT}$ & $\begin{array}{l}130 \text { parents of } \\
\text { children with minor } \\
\text { illnesses in ED }\end{array}$ & $\begin{array}{l}\text { Intervention: } 10-\text { min } \\
\text { video on paediatric } \\
\text { healthcare issues plus } \\
\text { information booklet on } \\
\text { common paediatric } \\
\text { ailments } \\
\text { Control: standard ED } \\
\text { discharge instructions }\end{array}$ & $\begin{array}{l}\text { Return visits to ED over } \\
6 \text { months }\end{array}$ & * \\
\hline
\end{tabular}




\begin{tabular}{|c|c|c|c|c|c|c|c|}
\hline Author(s)/Date & Setting & Aim & Design & Sample & Intervention & Main outcomes & $\begin{array}{l}\text { Quality } \\
\text { assessment* }^{*}\end{array}$ \\
\hline $\begin{array}{l}\text { Francis et al } \\
2009^{25}\end{array}$ & $\begin{array}{l}\text { General practice } \\
\text { (UK) }\end{array}$ & $\begin{array}{l}\text { Effect of interactive booklet } \\
\text { on respiratory tract } \\
\text { infections on reconsultation } \\
\text { for same illness episode, } \\
\text { antibiotic use, future } \\
\text { consultation intentions, and } \\
\text { parental satisfaction }\end{array}$ & Cluster RCT & $\begin{array}{l}61 \text { practices in } \\
\text { Wales and England. } \\
558 \text { parents of } \\
\text { children ( } 6 \text { months to } \\
14 \text { years) with a } \\
\text { respiratory tract } \\
\text { infection }\end{array}$ & $\begin{array}{l}\text { Intervention: Eight } \\
\text { page booklet on } \\
\text { childhood respiratory } \\
\text { tract infections within } \\
\text { consultations and as a } \\
\text { take home resource. } \\
\text { Control: 'usual' } \\
\text { consultation }\end{array}$ & $\begin{array}{l}\text { Reconsultation within } \\
2 \text { weeks, antibiotic } \\
\text { prescribing and } \\
\text { consumption, future } \\
\text { consultation intentions, } \\
\text { parent satisfaction and } \\
\text { usefulness of } \\
\text { information received, } \\
\text { reassurance and } \\
\text { enablement }\end{array}$ & 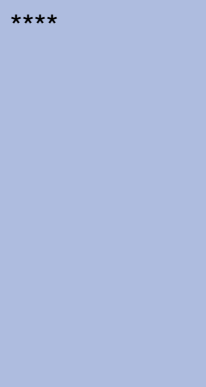 \\
\hline $\begin{array}{l}\text { Hansen } \\
1990^{26}\end{array}$ & $\begin{array}{l}\text { General practice } \\
\text { (Denmark) }\end{array}$ & $\begin{array}{l}\text { Effect of booklet on } \\
\text { families' minor } \\
\text { illness-behaviour for } \\
\text { children }<8 \text { years }\end{array}$ & $\mathrm{RCT}$ & $\begin{array}{l}100 \text { young families } \\
\text { with min. one child } \\
<8 \text { years in one } \\
\text { practice }\end{array}$ & $\begin{array}{l}\text { Intervention: Booklet } \\
\text { on common childhood } \\
\text { problems, presented by } \\
\text { GP. Parent recorded } \\
\text { illnesses. } \\
\text { Control: Unclear. } \\
\text { ?'usual care' plus diary } \\
\text { completion }\end{array}$ & $\begin{array}{l}\text { Consultation frequency } \\
\text { and anxiety over } \\
6 \text { months }\end{array}$ & $* *$ \\
\hline $\begin{array}{l}\text { McCarthy } \\
\text { et al } 1990^{23}\end{array}$ & $\begin{array}{l}\text { US Private } \\
\text { practice and } \\
\text { primary care } \\
\text { centre }\end{array}$ & $\begin{array}{l}\text { Effect of Acute Illness } \\
\text { Observation Scales (AIOS) } \\
\text { on mother's judgements } \\
\text { about acute illness in } \\
\text { children under } 24 \text { months }\end{array}$ & $\mathrm{RCT}$ & $\begin{array}{l}369 \text { mothers with } \\
\text { 2-week-old baby }\end{array}$ & $\begin{array}{l}\text { Intervention: AIOS film } \\
\text { plus fever scenario } \\
\text { scoring. Film shown } \\
\text { again at } 6 \text { and } \\
15 \text { months. AIOS used } \\
\text { to score illness prior to } \\
\text { and with doctor during } \\
\text { consultation. } \\
\text { Control: Routine advice } \\
\text { about fever. Illness } \\
\text { scored on 3-point scale }\end{array}$ & $\begin{array}{l}\text { Reliability, specificity } \\
\text { and sensitivity of } \\
\text { mother's judgements } \\
\text { compared to clinician } \\
\text { assessment from } \\
2 \text { weeks of age, for } \\
32 \text { months }\end{array}$ & * \\
\hline $\begin{array}{l}\text { Robbins et al } \\
2003^{12}\end{array}$ & $\begin{array}{l}\text { Primary care } \\
\text { (UK) }\end{array}$ & $\begin{array}{l}\text { Effect of home visit and } \\
\text { infant minor illness booklet } \\
\text { on parent's illness } \\
\text { management and } \\
\text { consultation rates }\end{array}$ & RCT & $\begin{array}{l}\text { Single GP practice: } \\
103 \text { parents of } \\
\text { babies born in } \\
6 \text {-month birth cohort }\end{array}$ & $\begin{array}{l}\text { Intervention: Postal } \\
\text { booklet on common } \\
\text { childhood illnesses. } \\
\text { Research nurse visit } \\
\text { when baby } 6 \text { weeks } \\
\text { old. } \\
\text { Control: Routine health } \\
\text { visiting service }\end{array}$ & $\begin{array}{l}\text { Confidence, knowledge, } \\
\text { home care activities and } \\
\text { desire to contact } \\
\text { professionals. } \\
\text { Prescription and } \\
\text { consultation rates } \\
\text { tracked for } 6 \text { months }\end{array}$ & $* * *$ \\
\hline $\begin{array}{l}\text { Thomson et al } \\
1999^{33}\end{array}$ & $\begin{array}{l}\text { General Practice } \\
\text { (UK) }\end{array}$ & $\begin{array}{l}\text { Effect of Baby Check, an } \\
\text { illness scoring system for } \\
\text { babies } \leq 6 / 12 \text {, on parents' } \\
\text { use of health services for } \\
\text { their baby }\end{array}$ & RCT & $\begin{array}{l}997 \text { mothers with } \\
\text { new babies }\end{array}$ & $\begin{array}{l}\text { Intervention: Baby } \\
\text { Check plus an accident } \\
\text { prevention leaflet } \\
\text { Control: accident } \\
\text { prevention leaflet alone }\end{array}$ & $\begin{array}{l}\text { Consultation behaviour } \\
\text { tracked for } 6 \text { months }\end{array}$ & 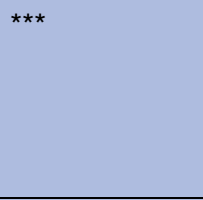 \\
\hline
\end{tabular}




\begin{tabular}{|c|c|c|c|c|c|c|c|}
\hline Author(s)/Date & Setting & Aim & Design & Sample & Intervention & Main outcomes & $\begin{array}{l}\text { Quality } \\
\text { assessment* }\end{array}$ \\
\hline $\begin{array}{l}\text { Usherwood } \\
1991^{35}\end{array}$ & $\begin{array}{l}\text { General practice } \\
\text { (UK) }\end{array}$ & $\begin{array}{l}\text { Effect of a children's } \\
\text { symptom booklet on GP } \\
\text { consultations }\end{array}$ & $\mathrm{RCT}$ & $\begin{array}{l}419 \text { households with } \\
634 \text { children born } \\
1975 \text { to } 1984 \\
\text { registered with one } \\
\text { practice }\end{array}$ & $\begin{array}{l}\text { Intervention: Postal } \\
\text { booklet on cough, } \\
\text { fever, sore throat, } \\
\text { diarrhoea and vomiting } \\
\text { Control: No } \\
\text { intervention. Baseline } \\
\text { data gathered for } \\
2 \text { months prior to } \\
\text { intervention }\end{array}$ & $\begin{array}{l}\text { Consultation rates for } \\
12 \text { months } \\
\text { postintervention }\end{array}$ & * \\
\hline \multicolumn{8}{|c|}{ Non-randomised trials } \\
\hline $\begin{array}{l}\text { Herman and } \\
\text { Jackson } \\
2010^{29}\end{array}$ & $\begin{array}{l}\text { Head Start } \\
\text { agencies (USA) }\end{array}$ & $\begin{array}{l}\text { Effect of educational } \\
\text { intervention on health } \\
\text { utilisation for acute illness } \\
\text { in children } \leq 5 \text { years }\end{array}$ & $\begin{array}{l}\text { Cohort study } \\
\text { (prospective) }\end{array}$ & $\begin{array}{l}9240 \text { parents with } \\
\text { one child enrolled in } \\
\text { Head Start } \\
7281 \text { completed the } \\
\text { training } \\
581 \text { tracked annually } \\
\text { for } 2 \text { years }\end{array}$ & $\begin{array}{l}\text { Health training } \\
\text { programmes using } \\
\text { reference guide 'What } \\
\text { to Do When Your Child } \\
\text { Gets Sick' by Mayer } \\
\text { and Kuklierus (2007) in } \\
55 \text { Head Start } \\
\text { agencies in } 35 \text { states. } \\
\text { Tracked for } 3 \text { months, } \\
\text { trained in } 4 \text { th month, } \\
\text { follow-up for } 6 \text { months. } \\
\text { Annual visits for } 581 \\
\text { parents }\end{array}$ & $\begin{array}{l}\text { ED and primary care } \\
\text { consultation rates for } \\
\text { 3-year period }\end{array}$ & *** \\
\hline $\begin{array}{l}\text { Isaacman } \\
\text { et al } 1992^{27}\end{array}$ & $\begin{array}{l}\text { Paediatric ED } \\
\text { (USA) }\end{array}$ & $\begin{array}{l}\text { Effect of two standardised } \\
\text { simplified discharge } \\
\text { instructions on parents } \\
\text { information recall }\end{array}$ & $\begin{array}{l}\text { CT (Non- } \\
\text { randomised } \\
\text { control) }\end{array}$ & $\begin{array}{l}197 \text { parents of } \\
\text { children discharged } \\
\text { with otitis media } \\
\text { (OM) }\end{array}$ & $\begin{array}{l}\text { Intervention 1: } \\
\text { standardised verbal } \\
\text { discharge information } \\
\text { on OM from HCPs in } \\
\text { ED } \\
\text { Intervention 2: as } \\
\text { above+typewritten } \\
\text { information from health } \\
\text { professionals in ED. } \\
\text { Control: 'usual' } \\
\text { discharge information }\end{array}$ & $\begin{array}{l}\text { Knowledge and } \\
\text { management of OM } \\
\text { before leaving ED, at } 24 \\
\text { and } 72 \mathrm{~h} \\
\text { postintervention } \\
\text { Return visits to ED and } \\
\text { parent reported } \\
\text { physician contact within } \\
72 \mathrm{~h}\end{array}$ & ** \\
\hline $\begin{array}{l}\text { Kelly et al } \\
1996^{36}\end{array}$ & $\begin{array}{l}\text { Private } \\
\text { paediatrician's } \\
\text { office, } 4 \text { Primary } \\
\text { care centres } \\
\text { (USA) }\end{array}$ & $\begin{array}{l}\text { Effect of educational } \\
\text { intervention on knowledge } \\
\text { and management of fever }\end{array}$ & $\begin{array}{l}\text { Pretest } \\
\text { post-test } \\
\text { cohort study }\end{array}$ & $\begin{array}{l}86 \text { caretakers of } \\
\text { children } 2 \text { months to } \\
5 \text { years presenting } \\
\text { for routine } \\
\text { healthcare or acute } \\
\text { minor illness } \\
50 \text { follow-up } \\
\text { interviews }\end{array}$ & $\begin{array}{l}\text { Printed fever } \\
\text { management sheet at } \\
\text { end of initial interview } \\
\text { Identified knowledge } \\
\text { deficits addressed }\end{array}$ & $\begin{array}{l}\text { Questionnaire on fever } \\
\text { knowledge and } \\
\text { management before and } \\
2-4 \text { weeks after } \\
\text { intervention }\end{array}$ & ** \\
\hline
\end{tabular}

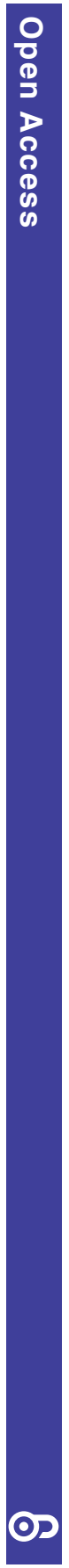

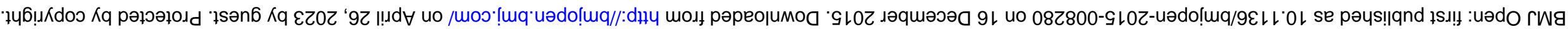




\begin{tabular}{|c|c|c|c|c|c|c|c|}
\hline Author(s)/Date & Setting & Aim & Design & Sample & Intervention & Main outcomes & $\begin{array}{l}\text { Quality } \\
\text { assessment }\end{array}$ \\
\hline $\begin{array}{l}\text { O'Neill } \\
\text { Murphy et al } \\
2001^{30}\end{array}$ & $\begin{array}{l}\text { Urban ED } \\
\text { Children's } \\
\text { Hospital of } \\
\text { Philadelphia } \\
\text { (USA) }\end{array}$ & $\begin{array}{l}\text { Effects of educational } \\
\text { programme on parents' } \\
\text { anxiety about fever, home } \\
\text { management and } \\
\text { consultation behaviour }\end{array}$ & $\begin{array}{l}\text { Quasi- } \\
\text { experimental, } \\
\text { pretest } \\
\text { post-test pilot } \\
\text { study }\end{array}$ & $\begin{array}{l}87 \text { parents with } \\
\text { children aged } \\
3 \text { months to } 5 \text { years } \\
\text { with fever }>38.4\end{array}$ & $\begin{array}{l}\text { Intervention: Interactive } \\
\text { Fever programme } \\
\text { Control: Standard } \\
\text { Fever Education } \\
\text { Programme }\end{array}$ & $\begin{array}{l}\text { Anxiety, consultation } \\
\text { behaviour, home } \\
\text { management before and } \\
\text { after HCP consultation, } \\
2 \text { and } 8 \text { weeks after the } \\
\text { intervention }\end{array}$ & * \\
\hline $\begin{array}{l}\text { Rosenberg } \\
\text { and Pless } \\
1993^{21}\end{array}$ & $\begin{array}{l}\text { Montreal } \\
\text { Children's } \\
\text { hospital ED } \\
\text { (Canada) }\end{array}$ & $\begin{array}{l}\text { Effect of ED-based parent } \\
\text { education on future ED } \\
\text { visit rates }\end{array}$ & $\begin{array}{l}\text { Non- } \\
\text { randomised } \\
\text { CT }\end{array}$ & $\begin{array}{l}300 \text { parents of } \\
\text { children }>6 \text { months } \\
\text { in ED }\end{array}$ & $\begin{array}{l}\text { Intervention: } \\
\text { educational pamphlet } \\
\text { on common childhood } \\
\text { illness plus video in } \\
\text { waiting room. } \\
\text { Control: usual' care. } \\
\text { (Sequential recruitment } \\
\text { to intervention then } \\
\text { control) }\end{array}$ & $\begin{array}{l}\text { Consultation behaviour } \\
4 \text { and } 12 \text { months } \\
\text { postintervention }\end{array}$ & \\
\hline $\begin{array}{l}\text { Steelman } \\
\text { et al } 1999^{22}\end{array}$ & $\begin{array}{l}\text { Military Paediatric } \\
\text { Clinic (USA) }\end{array}$ & $\begin{array}{l}\text { Effect of educational } \\
\text { intervention on parent's } \\
\text { childhood fever knowledge } \\
\text { and consultation rates }\end{array}$ & $\begin{array}{l}\text { Pretest } \\
\text { post-test CT }\end{array}$ & $\begin{array}{l}93 \text { parents attending } \\
2,4 \text {, and } 6 \text { month } \\
\text { well-infant visits }\end{array}$ & $\begin{array}{l}\text { Intervention: } \\
\text { standardised slide } \\
\text { presentation on } \\
\text { well-infant care+10 min } \\
\text { presentation on fever } \\
\text { and mail out at } 1 \text { and } \\
3 \text { months } \\
\text { Control: standardised } \\
\text { slide presentation on } \\
\text { well-infant care }\end{array}$ & $\begin{array}{l}\text { Knowledge of fever, } \\
\text { clinic and ED usage at } \\
\text { enrolment, } 2 \text { and } \\
4 \text { months } \\
\text { postintervention }\end{array}$ & \\
\hline $\begin{array}{l}\text { Wassmer and } \\
\text { Hanlon } \\
1999^{28}\end{array}$ & $\begin{array}{l}\text { Worcester Royal } \\
\text { Infirmary DGH } \\
\text { (UK) }\end{array}$ & $\begin{array}{l}\text { Effect of information for } \\
\text { parents on febrile } \\
\text { convulsions on parent's } \\
\text { knowledge }\end{array}$ & $\begin{array}{l}\text { Non- } \\
\text { Randomised } \\
\text { CT }\end{array}$ & $\begin{array}{l}\text { Intervention: } 50 \\
\text { parents of children } \\
\text { with } 1 \text { st febrile } \\
\text { convulsion May to } \\
\text { Dec } 1996 . \\
\text { Control: } 50 \text { parents } \\
\text { of children at } \\
\text { community health } \\
\text { clinic with no febrile } \\
\text { convulsion }\end{array}$ & $\begin{array}{l}\text { Intervention: verbal and } \\
\text { written information on } \\
\text { febrile convulsions } \\
\text { during consultation } \\
\text { Control: no information } \\
\text { provided. Assume } \\
\text { 'usual care' }\end{array}$ & $\begin{array}{l}\text { Parental knowledge of } \\
\text { febrile convulsion } 1 \text { year } \\
\text { postintervention }\end{array}$ & \\
\hline $\begin{array}{l}\text { Yoffe et al } \\
2011^{34}\end{array}$ & $\begin{array}{l}\text { Primary care } \\
\text { clinic (USA) }\end{array}$ & $\begin{array}{l}\text { Effect of parent-focused } \\
\text { educational intervention on } \\
\text { non-urgent ED visits }\end{array}$ & $\begin{array}{l}\text { Realistic } \\
\text { evaluation }\end{array}$ & $\begin{array}{l}\text { Parents of all } \\
\text { children } \leq 10 \text { years } \\
\text { attending } 3 \text { primary } \\
\text { care clinics } \\
\text { Number receiving }\end{array}$ & $\begin{array}{l}\text { Intervention: booklet on } \\
\text { common childhood } \\
\text { illness to the parents } \\
\text { with children registered } \\
\text { with one primary care } \\
\text { clinic }\end{array}$ & $\begin{array}{l}\text { ED consultation rates } \\
\text { Nov } 2007 \text { to Apr } 2009\end{array}$ & \\
\hline
\end{tabular}


Qualitative descriptive studies

Thornton et al Conducted in the $1991^{24}$ home (UK)

\section{Anhang et al Two Children's} $2013^{37} \quad$ EDs (USA)

Mixed methods studies

$\begin{array}{lll}\text { Stockwell } & \text { Early Head Start } \\ \text { et al } 2010^{31} & \text { Agency at } \\ & \text { Columbia } \\ & \text { University (USA) }\end{array}$

University (USA)

community-based,

culturally competent health

iteracy intervention on care

of URI, with Latino Early

Head Start parents

$\begin{array}{ll}\text { Design } & \text { Sample } \\ \text { Two booklet was not } & \begin{array}{l}\text { the bided } \\ \text { provils }\end{array} \\ & \begin{array}{l}\text { Study A: } 104 \\ \text { mothers of term } \\ \text { babies, randomly } \\ \text { selected from the } \\ \text { birth register } \\ \text { Study B: } 70 \text { mothers } \\ \text { of term babies born } \\ \text { on selected days }\end{array} \\ \end{array}$

Control: Parents of

children registered with

two other clinics not

receiving the booklet

Study A: Mothers used Views and use of the $\mathrm{BC}$ daily for a week and recorded contacts

with HCPs. Research nurse visit to grade

mother's competence

in booklet use

Study B: Mothers used

BC when wanted to

until baby was

6 months. Research

nurse visit when babies

8 and 16 weeks.

Questionnaire about

$\mathrm{BC}$ at 6 months

Pilot feasibility 294 parents/carers Intervention: Strategy

study of children for Off-site Rapid

Caregiver ratings of

usability of tool,

$\leq 18$ years who had

presented to an

emergency

sensitivity and specificity

tool web-based parent of SORT for Kids for

survey and severity identifying children

department for an

influenza-like illness

Pretest

11 parents of

scoring tool

needing ED

post-test pilot

children 6 months to modules delivered in

3 years in full

children's centre

Parental knowledge,

attitudes and care of

URI before and 2 weeks

after final module using

Knowledge, Attitude,

17 in interviews and

Practices instrument

${ }^{*}$ Quality assessment rating, between zero stars (lowest quality) and 4 stars (****, highest quality).

DGH, District General Hospital; ED, emergency department; GP, general practitioner; RCT/CT, randomised controlled trial/controlled trial; URI, upper respiratory infection. 
Table 2 Effectiveness of interventions on consultation rate

\begin{tabular}{|c|c|c|}
\hline Authors (date) & Consultation rate (significant results in bold) & Quality \\
\hline Anhang et al $2013^{37}$ & $\begin{array}{l}\text { The algorithm correctly classified } 93 \% \text { of paediatric patients with influenza-like illness who } \\
\text { made necessary ED visits and all children who made a second ED visit for influenza-like } \\
\text { illness within the subsequent week }\end{array}$ & * \\
\hline Baker et al $2009^{18}$ & No difference in reattendance to ED. $p=0.4695 \% \mathrm{Cl}-0.06$ to 0.16 & *** \\
\hline Chande et al $1996^{20}$ & No difference in contact with primary care physician $(p=0.37)$ or return visits to ED $(p=0.68)$ & * \\
\hline Francis et al $2009^{25}$ & $\begin{array}{l}\text { Non-significant reduction in reconsultation in first } 2 \text { weeks } \\
p=0.2995 \% \mathrm{Cl}-2.7 \text { to } 9.3 \\
\text { Significant reduction in intention to consult in future for similar illness }(55.3 \% \text { intervention vs } \\
76.4 \% \text { control) } \mathbf{p}<0.001 \mathrm{Cl} 0.20 \text { to } 0.57\end{array}$ & 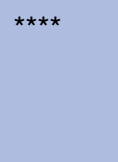 \\
\hline Hansen $1990^{26}$ & $\begin{array}{l}\text { Reported significant reduction in consultations in intervention group (mean consultations } \\
0.288 \text { (2SD } 0.315-0.252) \text { intervention vs } 0.426(0.461-0.390) \text { control group). p Value not } \\
\text { given but states as significant }\end{array}$ & ** \\
\hline Herman and Jackson & Significant reduction in choosing to contact HCP first. Pre $69 \%$ Post $33 \% \mathbf{p}<0.0001$ & $\star \star \star *$ \\
\hline $2010^{29}$ & $\begin{array}{l}\text { Significant reduction in ED (by } 58 \% \mathbf{p}<0.00195 \% \mathrm{Cl} 0.51 \text { to } 0.50 \text { ) and doctor visits (by } \\
42 \% \mathbf{p}<0.00195 \% \mathrm{Cl} 0.33 \text { to } 0.46 \text { ) }\end{array}$ & \\
\hline Isaacman et al $1992^{27}$ & $\begin{array}{l}\text { Parent reported physician contact showed a non-significant reduction ( } 22.8 \% \text { control vs } \\
13.2 \% \text { intervention group). Return to ED rates by day } 3 \text { were significantly reduced in } \\
\text { intervention groups ( } 3.1 \% \text { intervention vs } 10.1 \% \text { control group } p=0.05)\end{array}$ & ** \\
\hline Kai $1994^{32}$ & $\begin{array}{l}14 \text { parents reported that on } 19 \text { occasions Baby Check influenced their decision not to } \\
\text { contact a doctor }\end{array}$ & ** \\
\hline $\begin{array}{l}\text { O'Neill Murphy et al } \\
2001^{30}\end{array}$ & High attrition to follow-up resulted in no data on effect on consultation rate & * \\
\hline Robbins et al $2003^{12}$ & $\begin{array}{l}\text { Significant reduction in visits to child health clinic (median visits: intervention } 4.5 \text { vs control } \\
5 \mathrm{p}=\mathbf{0 . 0 3 9 )} \\
\text { No significant difference in GP, HV or minor illness nurse contacts }\end{array}$ & *** \\
\hline Rosenberg and Pless & Non-significant reduction in ED use in intervention group. Mean total medical visits/year: & \\
\hline Steelman et al $1999^{22}$ & $\begin{array}{l}\text { po significant differences in clinic or ED use between control and intervention groups, but } \\
\text { group }=5 \text { 'inappropriate' visits, intervention group }=7 \text { such visits vs } 1 \text { 'inappropriate' visit for } \\
\text { both intervention and control in families with } 1 \text { child only } p=0.04)\end{array}$ & \\
\hline Thomson et al $1999^{33}$ & $\begin{array}{l}\text { No significant difference in total consultations } p=0.26 \text {, GP } p=0.30 \text {, out of hours service use } \\
p=0.93 \text { or referrals } p=0.64\end{array}$ & $* * *$ \\
\hline Usherwood $1991^{35}$ & $\begin{array}{l}\text { No significant difference was found in the number of daytime health centre contacts } \\
\text { Significant decrease in home visits in the intervention group for households with one or two } \\
\text { children }(28 \% \text { reduction, } p<0.05 \text { ) but not for larger families } \\
\text { Significant increase in out of hours contacts in the intervention group (mean contacts: } 1 \\
\text { child family Control } 0.03 \text { vs Intervention } 0.10 ; 2 \text { child } C: 0.11 \text { vs I:0.23; } 3 \text { child } C: 0.06 \text { vs } \\
\text { I: } 0.30 p<0.05)\end{array}$ & * \\
\hline Yoffe et al $2011^{34}$ & $\begin{array}{l}\text { Statistically significant reduction in ED use in intervention group } \mathbf{p}<0.001 \text {. Reductions } \\
\text { ranged from } 55 \text { to } 81 \% \text { compared to the same month in the previous year }\end{array}$ & \\
\hline Summary & $\begin{array}{l}6 / 15 \text { studies significant difference including } 1 \text { reduction in intention to consult, } 1 \text { reduction in } \\
\text { home visits but with increase in out of hours services } \\
\text { Quality assessment rating between zero stars (lowest) quality and four stars (highest) }\end{array}$ & \\
\hline
\end{tabular}

found that parents felt 'Baby Check' had increased their confidence to monitor their child and given them 'moral support' for their decision to consult a doctor.

\section{Antibiotic prescription}

Four studies assessed the effect of interventions on antibiotic prescription. Francis et $a l^{25}$ (high quality) found a significant reduction in In antibiotic prescriptions given by clinicians in the intervention group (19.5\% intervention vs $40.8 \%$ control (95\% CI 13.7 to $28.9, \mathrm{p}<0.001)$ ); and Stockwell et $a l^{\beta 1}$ showed a reduction in the number of parents who sought antibiotics without a prescription or used over the counter medication inappropriately; however this small study (11 parents) failed to report effects on antibiotics sought by parents from health professionals. Two other studies (both high quality) ${ }^{12} 33$ found no significant differences in antibiotic prescribing.

Factors influencing the effectiveness of an intervention

Factors which may have influenced the effectiveness of interventions were identified from a comparison of study populations and/or the setting of the study and 
Table 3 Effectiveness of interventions on parents' knowledge

\begin{tabular}{|c|c|c|}
\hline Author (date) & Parent's knowledge (significant results in bold) & Quality \\
\hline Baker et al $2009^{18}$ & $\begin{array}{l}\text { Significant reduction in knowledge scores: } 54 \% \text { reduction in responses that fever was } \\
\text { dangerous ( } p<0.0001,95 \% \mathrm{Cl} 0.43 \text { to } 0.65) 28 \% \text { reduction in responses that child with } \\
\text { fever should be woken }(p<0.0001,95 \% \mathrm{Cl} 0.19 \text { to } 0.39) 30 \% \text { increase in responses } \\
\text { identifying aspirin as inappropriate }(\mathbf{p}<0.0001,95 \% \mathrm{Cl}-0.42 \text { to } 0.16)\end{array}$ & *** \\
\hline Broome et al $2003^{19}$ & $\begin{array}{l}\text { Knowledge increased significantly more in both groups than in control group at } 24-72 \mathrm{~h} \text { and } \\
1,3 \text { and } 6 \text { months } p<0.03 \text {. No information on the size of the effect provided. Those given } \\
\text { individual instruction reported to have higher scores-no } p \text { value provided }\end{array}$ & * \\
\hline Isaacman et al $1992^{27}$ & 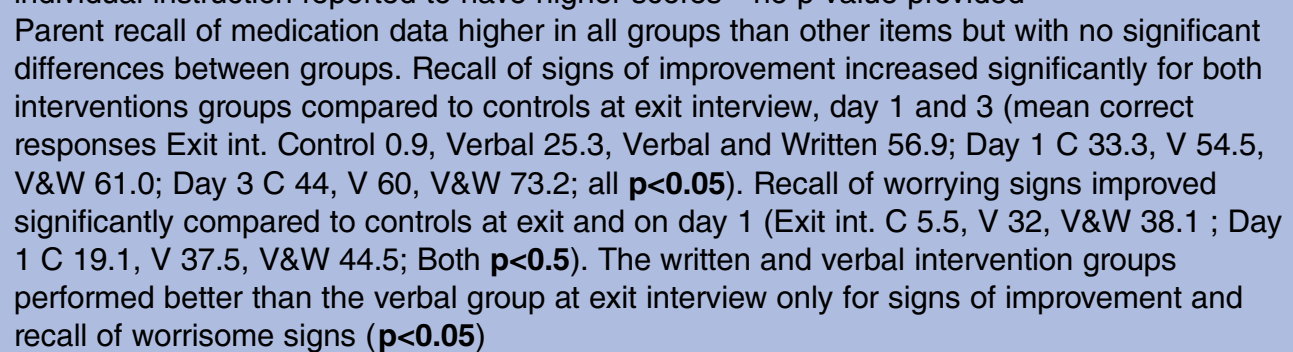 & ** \\
\hline Kelly et al $1996^{36}$ & $\begin{array}{l}\text { Indirect measurement of knowledge: } \\
\text { No significant difference in level of fever at which antipyretics were administered } \\
(p=0.91) \text {. A significant difference was found in accuracy of antipyretic dose }(n=30 \\
\text { incorrect dose preintervention, } 18 / 30(60 \%) \text { accurate doses postintervention } p=0.04)\end{array}$ & ** \\
\hline McCarthy et al $1990^{23}$ & $\begin{array}{l}\text { Indirect measurement of knowledge: } \\
\text { Reliability of mother's judgements: intervention group were more likely to agree with } \\
\text { clinician than control group: } 91.7 \% \text { vs } 72.4 \% \text { ( } \kappa 0.50 \text { vs } 0.26 \text { ) } \\
\text { specificity of mother's judgements: Mothers in the intervention group were less likely to } \\
\text { group (Intervention } 90 \% \text { vs } 59 \% \text { control group p<0.0001) } \\
\text { Sensitivity of mother's judgements: Serious illness was the outcome used to measure } \\
\text { sensitivity. No difference found between intervention and control group ( } 80 \% \text { vs } 90 \% \\
\text { respectively) }\end{array}$ & * \\
\hline Robbins et al $2003^{12}$ & Non-significant reduction in knowledge at 7 months in intervention group & *** \\
\hline Steelman et al $1999^{22}$ & $\begin{array}{l}\text { Significantly fewer incorrect responses in intervention group at } 2 \text { months (Intervention } 10.4 \\
\text { vs Control } 11.8 ; p=0.006 \text { ) and at } 4 \text { months (Intervention } 8.5 \text { vs Control } 10.3 ; p=0.002 \text { ) }\end{array}$ & \\
\hline Stockwell et al $2010^{31}$ & Significant increase in knowledge/attitude health literacy score $(61 \% \mathbf{p}<0.05)$ & ** \\
\hline $\begin{array}{l}\text { Wassmer and Hanlon } \\
1999^{28}\end{array}$ & $\begin{array}{l}\text { Significant increase in parental knowledge of febrile convulsion in the intervention group } \\
\mathbf{p}<0.05 \text { but these parents children had already had a febrile convulsion. See the original } \\
\text { paper for details on size of the effect as these are reported per question asked of parents }\end{array}$ & \\
\hline Summary & $\begin{array}{l}8 / 9 \text { showed significant increase in knowledge, although implied in } 2 \text { studies and } 1 \text { study had } \\
\text { high risk of bias. } 1 \text { paper showed reduction in knowledge at } 7 \text { months. } 1 \text { qualitative paper }\end{array}$ & \\
\hline
\end{tabular}

the content, format and delivery of the educational interventions.

\section{Content of interventions: range of topics addressed by the interventions}

Eleven studies assessed interventions which focused on a single symptom or type of childhood illness alone (such as fever, febrile convulsions, respiratory tract infection, otitis media), while 10 provided information on a range of different childhood illnesses.

Three single-topic studies measured consultation behaviour, of which Francis $e t a l^{25}$ found reduced intention to consult in the intervention compared to control group while two did not. ${ }^{18} 22$ Two single-topic studies assessed anxiety/reassurance, one found no effect ${ }^{25}$ and the other a reduction in intervention and control groups. ${ }^{30}$ Confidence was assessed in one single-topic study ${ }^{19}$ which found no effect. Antibiotic prescribing was assessed in two respiratory focused studies, ${ }^{25}{ }^{31}$ one of which showed a significant reduction in prescribing in the intervention group in the first 2 weeks postintervention $^{25}$ and the other a non-significant reduction in seeking antibiotics without prescription after the intervention $^{31}$ (only Francis et al studied rated as high quality).

Four of the 10 studies evaluating the effects of providing information on multiple childhood illnesses or symptoms showed trends towards reduction in consultation rates or intention to consult ${ }^{26} 293435$ (one high-quality). Four multitopic intervention studies reported a reduction in anxiety or increased reassurance ${ }^{24} 262932$ (one high quality). Confidence improved in two of the 'Baby Check' studies $^{24}{ }^{32}$ (one high quality) but in another (high-quality) study, there was no effect on confidence. ${ }^{12}$ 
Table 4 Effectiveness of interventions on parents' anxiety of reassurance

\begin{tabular}{|c|c|c|}
\hline Author (date) & Anxiety/reassurance (significant results in bold) & Quality \\
\hline Francis et al $2009^{25}$ & No significant difference in level of reassurance & 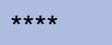 \\
\hline Hansen $1990^{26}$ & $\begin{array}{l}\text { Significant reduction in worry reported as the main reason for consulting the GP }(19 \% \text { vs } \\
31 \% p=0.0075)\end{array}$ & ** \\
\hline $\begin{array}{l}\text { Herman and Jackson } \\
2010^{29}\end{array}$ & $\begin{array}{l}\text { Parents reporting being 'very worried' when their child is sick reduced by a third (no further } \\
\text { statistics available) }\end{array}$ & *** \\
\hline Kai $1994^{32}$ & $\begin{array}{l}11 \text { parents consulted despite low acuity scores to avoid consulting later 'out of hours', or } \\
\text { because they wanted reassurance } \\
\text { Baby Check did not answer their questions or tell them how to manage minor illness }\end{array}$ & ** \\
\hline Krantz $2001^{38}$ & $\begin{array}{l}\text { Parents felt that the fever guide was reassuring and that the decision guide on what to do } \\
\text { when was important to include }\end{array}$ & * \\
\hline $\begin{array}{l}\text { O’Neill Murphy et al } \\
2001^{30}\end{array}$ & At 2 weeks both groups were less anxious. Control $86 \%$ Intervention $50 \%$ & * \\
\hline Thornton et al $1991^{24}$ & $\begin{array}{l}\text { In the first part of the study } 46 \% \text { found using Baby Check reassuring and } 4 \% \text { said it caused } \\
\text { anxiety. } 6 \% \text { of mothers reported that Baby Check helped them to decide whether or not to } \\
\text { seek advice, } 4 \% \text { were reassured by a low score. Two with high scores were prompted to } \\
\text { seek help }\end{array}$ & $\star \star \star \star *$ \\
\hline Summary & $\begin{array}{l}1 / 7 \text { significant reduction in worry. } 3 \text { reduced anxiety but descriptive statistics only. } 2 \\
\text { qualitative papers }\end{array}$ & \\
\hline
\end{tabular}

Neither of two high-quality multitopic studies demonstrated a significant reduction in antibiotic prescribing. ${ }^{12} 33$

In summary, reduction in consultation rates, reduction in anxiety and increases in confidence appeared more common in multitopic compared to single-topic interventions, while reduction in antibiotic prescribing was more effective with single illness-focused interventions.

\section{Content of interventions: information on assessment and/or management of childhood illness}

Four interventions specifically intended to enable parents to assess the severity of their baby's illness and know when to seek medical attention for their child $^{23243233}$ (two high quality). One of these interventions (a low quality study) informed parents about fever and home management of fever and found that $90 \%$ of parents rated the information helpful in decisionmaking and as a communication tool. ${ }^{19}$ In contrast, nearly one-third of parents did not think the 'Baby Check' educational tool was useful, ${ }^{24}$ and a qualitative study of the same tool ${ }^{32}$ revealed that even when parents scored their child's illness as minor they still consulted for the illness within $24 \mathrm{~h}$ after the assessment, because they wanted practical advice on management.

\section{Content of the interventions: accessibility of the information}

Many of the papers provided brief descriptions of the strategies used to make interventions easy to understand for parents. Three (one high quality) designed their interventions specifically for parents with low levels of health literacy. ${ }^{29} 3138$ The language used in the 'Baby Check' score card was simplified to accommodate low health literacy through the translation of professional terms such as 'reduced tone' as 'floppiness' ${ }^{24}$ and a further three studies reported that their interventions were designed for age 11-12-year-old reading level. ${ }^{30} 34{ }^{39}$ One study specifically mentioned using cartoons and humour to increase the accessibility of information. ${ }^{34}$ There was no identifiable relationship on outcomes between studies which did or did not design interventions for easy reading. However, Krantz's ${ }^{38}$ qualitative study evaluating parents' views of a fever guide found that parents liked the one page, easy-to-read style, the use of simple diagrams such as a thermometer showing both Fahrenheit and Celsius and pictures of how to measure a child's temperature. Parents felt that these pictures were likely to enhance recall of the information.

\section{Delivery method for interventions: interactive or one-way flow}

Six studies provided educational interventions to parents in an interactive manner, that is, the parent could engage with the intervention rather than just receiving information: ${ }^{19} 2325 \quad 29-31 \quad 36$ two (high-quality studies) showed significant reductions in consultation rates or intention to consult ${ }^{2529}$ and four significantly improved parental knowledge 233136 (low to $2 *$ quality).

Two additional but low to $2^{*}$ quality studies ${ }^{19}$ used a relatively simple non-discursive method to provide information to parents, showing significant reductions in consultations of up to $88 \%$ in a comparison of attendances to an emergency department per month 1 year following the intervention. These shared a common feature: when health professionals gave their booklets to parents, they emphasised that the content was important and would help them to look after their acutely sick child. These findings intimate that educational interventions 
can be successful even when they are provided using a simple method, but clearly further studies are needed to demonstrate this.

\section{Intervention setting}

None of the four interventions which were delivered in the waiting room of an emergency department ${ }^{18} 202130$ (one high quality) had significant effects on consultation rates, anxiety or parental knowledge. These studies involved both single topic and multitopic interventions with varying delivery mechanisms and suggest that it is the environment in which the intervention was delivered which is associated with effectiveness, rather than the content of the intervention itself.

Two US studies ${ }^{29} 31$ took place in children's health centres: one high-quality study reduced consultation rates in local emergency departments and primary care $^{29}$ and the other improved parental knowledge. ${ }^{31}$ Peer support and a trustworthy environment were two important factors suggested by the authors as related to this success.

\section{Parent involvement in intervention development or evaluation}

One high-quality study involved parents in the development $^{25}$ and four in the evaluation of the educational intervention. ${ }^{192629} 35$ Four showed reduction in consultation rates, intention to consult, or improved parental knowledge. ${ }^{19} 2526{ }^{29}$ In comparison, studies using existing educational materials as their intervention, without modification and evaluation by its target population, were less successful ${ }^{12} 33$ (both high quality).

\section{DISCUSSION}

This systematic review and synthesis of information resources intending to help parents decide when to seek medical help for an acutely sick child identified measures of effectiveness used to evaluate interventions, as well as factors which appear to influence the effectiveness of interventions. Unlike previous reviews which focused on interventions specifically for respiratory tract infections ${ }^{40}$ or acute paediatric hospital admissions, ${ }^{41}$ our review was broader as we identified factors influencing effectiveness of interventions on parents' help-seeking behaviour for all common acute illnesses at home.

\section{Measures of effectiveness}

Consultation frequency, knowledge, reassurance/ anxiety, satisfaction, confidence and antibiotic prescribing were used as measures of effectiveness. Studies which found reductions in consultation $\operatorname{rates}^{27} \quad 29 \quad 34$ were all conducted in the USA, which may reflect differences in health service delivery systems and possible financial costs associated with unscheduled consultations. These differences in parental motivations may limit applicability in other countries such as the UK where direct parent-incurred health service costs are less relevant.
Results from studies measuring parents' knowledge of acute childhood illness indicate that when both verbal and written information were provided, parents were more likely to retain knowledge in the long term than when only given written information. ${ }^{19} 22 \quad 23 \quad 283133 \quad 37$ Verbal reinforcement may signal to parents that health professionals endorse the information.

Providing information did not seem to be directly linked to increased satisfaction, although it is not clear whether the studies we found used a valid measurement tool. Limited information was available about the methods used to measure parent satisfaction, which included a question over the phone, ${ }^{27}$ or using one or two items within a rating scale administered by phone. ${ }^{1925}$ Satisfaction is a complex phenomenon and it is therefore unlikely that such simple measures will elucidate factors which influence it. No conclusions can be drawn regarding the impact of interventions on parents' confidence to care for their child.

The effectiveness of interventions at reducing antibiotic prescriptions mirror those of Andrews et $a l_{\mathrm{s}}{ }^{40}$ review of interventions specifically focused on reducing consultation and antibiotic use in respiratory tract infection, which found that educational materials reduced consultation rates by up to $40 \%$. The two respiratory focused studies which we identified, one from the UK and one from the USA, both indicated a reduction in antibiotic use, while neither of the less focused interventions demonstrated any effect on antibiotic use.

We were unable to easily identify an intervention which works consistently to reduce consultation rates, to improve parents' knowledge, confidence or satisfaction.

\section{Factors influencing the effectiveness of an intervention}

Interventions providing information on multiple childhood illnesses or symptoms appeared to be more effective (eg, reduction in consultation rates or intention to consult, reduction in anxiety or increased reassurance), compared to interventions addressing single symptoms. This may be because common childhood symptoms, such as fever, cough, sore throat, vomiting and diarrhoea, often occur simultaneously. Therefore, although parents receiving fever education may feel more competent in managing fever, they may continue to seek a medical consultation for other symptoms about which they have less knowledge or confidence. Moreover, educational material which addressed the assessment of illness severity as well as management of minor illness appear to be more effective in supporting parents to care for their children and seek help when necessary: if information is only provided on assessment this may still leave parents needing advice about how to manage, even minor, illness.

Parents' involvement in the development of educational interventions may improve effectiveness. These findings support the general trend towards involving patients and the public in research, ${ }^{4}$ emphasising the 
importance of working collaboratively with the end users of interventions.

O'Neill-Murphy et $a l^{30}$ argued that information provided in an interactive method is more effective in improving knowledge than non-interactive methods. However, our findings do not clearly support this position as we noted significant effects for interventions delivered with, and without, interaction. Involving health professionals in the distribution of booklets, with or without an interactive discussion, may increase the perceived value and reliability of the information and motivate parents to read the booklets, trust the home management strategies suggested and, finally, impact on their behaviour. Parents have previously been found to trust information from doctors more than that from other sources. ${ }^{9}$

Studies in the review were conducted in a range of settings; those conducted in emergency departments were the least effective. ${ }^{18} 202130$ Having an acutely sick child is a stressful time for parents, generating considerable anxiety and uncertainty about when to seek medical help. ${ }^{59}{ }^{11}$ Stress can impair learning, ${ }^{42}{ }^{43}$ therefore it is not surprising that in Chande et als study only $65 \%$ of participants in the intervention group remembered the video in the emergency department. However, two US studies $^{29} 31$ conducted in children's health centres showed reduction in consultation rates in local emergency departments and in primary care ${ }^{29}$ and improved parental knowledge. ${ }^{31}$ We do not know whether interventions delivered in children's centres would similarly work in the UK, although community education on childhood illness has been suggested in a recent UK survey of parents' first contact choices. ${ }^{43}$

\section{Strengths and limitations}

The strengths of our review lie in its inclusiveness. Given the non-comparable research designs, we used an integrative narrative approach, recognised as an effective method for summarising and synthesising findings across multiple study designs. ${ }^{16}{ }^{17}$ This approach enabled us to identify influences on effectiveness across a wider range of studies and topics than would have been possible with a single study type or topic focused review. This comprehensive strategy does result in the inclusion of low quality studies whose impact may be questioned and means our recommendations need to confirmed in further studies.

It is possible some studies were missed as the screening of titles and abstracts for inclusion was performed by only one person. The highly heterogeneous nature of the included studies in terms of design, as well as interventions, outcomes measured, populations and settings limited our ability to perform more quantitative syntheses. The literature search was of papers published in English since January 1990. However, it was evident that some of the earlier included studies are already of limited direct relevance to contemporary health services. For example, the 'Baby Check' tool used in three studies included a requirement for parents to measure rectal temperature, which is no longer recommended practice. Also no studies compared differing healthcare delivery systems; health systems are likely to have implications on the impact of different interventions.

\section{Recommendations for clinical practice: how best to} provide information to help parents decide when to seek help for an acutely sick child

Our findings indicate that interventions with the following characteristics are more likely to be effective:

- Comprehensive information on childhood illness;

- Information on assessment of children's need for a medical consultation and on how to manage minor illness at home;

- Reinforcement or support by local healthcare professionals;

- Delivery away from the stressful environment of the emergency department. This could be in primary care, in the home or in social care settings;

- Coproduction with parents.

Even without the development of new materials for parents of acutely ill children, there are messages here for clinicians using existing materials. Clinicians need to select resources which provide information on multiple common symptoms of childhood illness. Evidence from focus groups parents indicates development with parents is good practice. Interventions in this area can have unexpected consequences which need to be considered prior to implementation, as, for example, one primary care-based intervention which resulted in shifting consultation from day time home visits to the out of hours service. $^{35}$

Information is best provided in primary care or social care settings. Community centres such as SureStart Children's Centres in the UK provide a potential route for the delivery of health information by health professionals, such as health visitors.

\section{Directions for future research}

Most of the studies included in the review were quantitative, providing valuable information on the effects of educational interventions. More qualitative studies are needed, which are able to provide in-depth understanding about what, how and why interventions affect parents' abilities to assess and manage acute childhood illnesses. This information should be underpinned by research which identifies both parents' and health professionals' current use of information resources, and their views on how these resources need to be developed. Finally it is important that any future interventions for parents should be co-developed with parents themselves. ${ }^{44}$ Given the rising rates of consultations and the considerable impact this is having on the health service in the UK, as well as on parents, there is a pressing need for larger scale implementation studies taking into account the findings of this review. 


\section{CONCLUSION}

Overall, the majority of reviewed interventions had limited effects on consultation rates. Although many studies showed an improvement in parental knowledge of childhood illness, this did not necessarily lead to more confidence and less anxiety in parents when looking after their child at home. Interventions providing comprehensive information on childhood illness which can be used for both assessing children's need for a medical consultation and for managing minor illness at home were more effective in reducing consultation rates than those focused on a single symptom/illness or only on assessing the child's level of acuity. Interventions also appeared more effective if parents were involved in their development or evaluation.

\section{Author affiliations}

${ }^{1}$ School of Health, University of Northampton, Northampton, UK

${ }^{2}$ Sapphire Group, Health Sciences, University of Leicester, Leicester, UK

${ }^{3}$ Paediatric Emergency Medicine Leicester Academic (PEMLA) Group, Leicester Hospitals, Leicester, UK

${ }^{4}$ Nuffield Department of Primary Care Health Sciences, University of Oxford, Oxford, UK

${ }^{5}$ Department of Family Medicine, University of Washington, Seattle, USA ${ }^{6}$ Department of Population, Policy and Practice, Institute of Child Health, University College London, London, UK

Twitter Follow Damian Roland at @damian_roland and Sarah Neill @SarahNeill7

Acknowledgements The authors thank Dr Chenyu Shang, for the early work searching and reviewing the literature.

Contributors SN, ML, CHDJ and MT conceived the original idea. Initial data searching was performed by SN and quality analysis undertaken by all authors. SN prepared an initial manuscript which DR revised. All authors contributed to the final version.

Funding This publication presents independent research funded by the University of Leicester. The views expressed in this publication are those of the author(s) and not necessarily those of the University of Leicester. MT and CHDJ: This report is independent research arising from MT's Career Development Fellowship supported by the National Institute for Health Research. The views expressed in this publication are those of the author(s) and not necessarily those of the NHS, the National Institute for Health Research or the Department of Health.

Competing interests None declared.

Provenance and peer review Not commissioned; externally peer reviewed.

Data sharing statement No additional data are available.

Open Access This is an Open Access article distributed in accordance with the terms of the Creative Commons Attribution (CC BY 4.0) license, which permits others to distribute, remix, adapt and build upon this work, for commercial use, provided the original work is properly cited. See: http:// creativecommons.org/licenses/by/4.0/

\section{REFERENCES}

1. Kennedy I. Getting it right for children and young people. Overcoming cultural barriers in the NHS so as to meet their needs. A review by Professor Sir lan Kennedy September 2010. London: Department of Health, 2010.

2. Hippisley-Cox J, Vinogradova $Y, Q$ Research. Trends in Consultation Rates in General Practice 1995/1996 to 2008/2009: Analysis of the QResearch $®$ database.Final Report to the NHS Information Centre and Department of Health. Nottingham: NHS The Information Centre for Health and Social Care, University of Nottingham, 2009.

3. Royal College of General Practitioners. Weekly Returns Service Annual Prevalence Report 2007. Birmingham: Royal College of General Practitioners, Birmingham Research Unit, 2007.

4. Carson D, Clay H, Stern R. Primary care and emergency departments. Report from the Primary Care Foundation. Primary Care Foundation, March 2010. http://www.primarycarefoundation.co. uk/index.html

5. Tadros S, Wallis D, Sharland M. Lack of use for advice by parents results in increasing attendance to the paediatric emergency department. Arch Dis Child 2009;94:483.

6. Thompson C, Hayhurst C, Boyle A. How have changes to out-of-hours primary care services since 2004 affected emergency department attendances at a UK District General Hospital? A longitudinal study. Emerg Med J 2009;27:22-5.

7. Maguire S, Ranmal R, Komulainen $\mathrm{S}$, et al. Which urgent care services do febrile children use and why? Arch Dis Child 2011;96:810-16.

8. Williams A, O'rourke P, Keogh S. Making choices: why parents present to the emergency department for non-urgent care. Arch Dis Child 2009;94:817-20.

9. Neill SJ. Family management of acute childhood illness at home: a grounded theory study, in Nightingale school of nursing and midwifery. London: King's College London, 2008.

10. Kai J. Parents difficulties and information needs in coping with acute illness in preschool children: a qualitative study. BMJ 1996;313:987-90.

11. Houston AM, Pickering AJ. 'Do I don't I call the doctor': a qualitative study of parental perceptions of calling the GP out-of-hours. Health Expect 2000;3:234-42.

12. Robbins H, Hundley V, Osman LM. Minor illness education for parents of young children. Int J Nurs Stud 2003;44:238-47.

13. Heaney D, Wyke S, Wilson $P$, et al. Assessment of impact of information booklets on use of healthcare services: randomised controlled trial. BMJ 2001;322:1218-21.

14. Neill SJ. Acute childhood illness at home: the parents perspective. J Adv Nurs 2000;31:821-32.

15. Pluye $\mathrm{P}$, Robert $\mathrm{E}$, Cargo $\mathrm{M}$, et al. Proposal: a mixed methods appraisal tool for systematic mixed studies reviews. http://www. webcitation.org/5tTRTc9yJ, 2011 Sept 2013; http://mixedmethod sappraisaltoolpublic.pbworks.com

16. Popay J, Roberts H, Sowden A, et al. Guidance on the conduct of narrative synthesis in systematic reviews. A product from the ESRC methods programme. Lancaster: Lancaster University, 2006.

17. Dixon-Woods M, Agarwal S, Young B, et al. Integrative approaches to qualitative and quantitative evidence. London: NHS Health Development Agency, 2004.

18. Baker M, Monroe KW, King WD, et al. Effectiveness of fever education in pediatric emergency department. Pediatr Emerg Care 2009;25:565-8

19. Broome M, Dokken DL, Broome CD, et al. A study of parent/ grandparent education for managing a febrile illness using the CALM approach. J Pediatr Health Care 2003;17:176-83.

20. Chande V, Wyss N, Exum V. Educational interventions to alter pediatric emergency department utilisation patterns. Arch Pediatr Adolesc Med 1996;150:525-8.

21. Rosenberg E, Pless I. Can effective parent education occur during emergency room visits? Fam Med 1993;25:598-601.

22. Steelman J, Kotchmar GS Jr, Brehm WT, et al. Childhood fever education in a military population: is education enough? J Miss State Med Assoc 1999;40:407-9.

23. McCarthy $\mathrm{P}$, Sznajderman SD, Lustman-Findling K, et al. Mothers' clinical judgement: a randomized trial of the Acute Illness Observation Scales. J Pediatr 1990;116:200-6.

24. Thornton AJ, Morley CJ, Green SJ, et al. Field trials of the Baby Check score card: mothers scoring their babies at home. Arch Dis Child 1991;66:106-10.

25. Francis NA, Butler CC, Hood K, et al. Effect of using an interactive booklet about childhood respiratory tract infections in primary care consultations on reconsulting and antibiotic prescribing: a cluster randomised controlled trial. BMJ 2009;339:b2885.

26. Hansen B. A randomised controlled trial on the effect of an information booklet for young families in Denmark. Patient Educ Couns 1990;16:147-50.

27. Isaacman D, Purvis K, Gyuro J, et al. Standardised instructions: do they improve communication of discharge information from the emergency department? Pediatrics 1992;89:1204-8.

28. Wassmer E, Hanlon M. Effects of information on parental knowledge of febrile convulsions. Seizure 1999;8:421-3. 
29. Herman A, Jackson P. Empowering low-income parents with skills to reduce excess pediatric emergency room and clinic visits through a tailored low literacy training intervention. J Health Commun 2010;15:895-910.

30. O'Neill-Murphy K, Liebman M, Barnsteiner J. Fever education: does it reduce parent fever anxiety? Pediatr Emerg Care 2001;17:47-51.

31. Stockwell M, Catallozzi M, Meyer D, et al. Improving care of upper respiratory infections among Latino Early Head Start parents. J Immigr Minor Health 2010;12:925-31.

32. Kai J. 'Baby Check' in the inner city-use and value to parents. Fam Pract 1994;11:245-50.

33. Thomson $\mathrm{H}$, Ross $\mathrm{S}$, Wilson $\mathrm{P}$, et al. Randomised controlled trial of effect of Baby Check on use of health services in first 6 months of life. BMJ 1999;318:1740-4.

34. Yoffe S, Moore RW, Gibson JO, et al. A reduction in emergency department use by children from a parent educational intervention. Fam Med 2011;43:106-11.

35. Usherwood T. Development and randomised controlled trial of a booklet of advice for parents. Br J Gen Pract 1991;41:58-62.

36. Kelly L, Morin K, Young D. Improving caretakers' knowledge of fever management in preschool children: is it possible? $J$ Pediatr Health Care 1996;10:167-73.

37. Anhang R, Fagbuyi D, Harris R, et al. Feasibility of web-based self-triage by parents of children with influenza-like illness. JAMA Pediatr 2013;167:112-18.
38. Krantz C. Childhood fevers: developing an evidence-based anticipatory guidance tool for parents. Pediatr Nurs 2001;27: 567-71.

39. Francis N, Wood F, Simpson S, et al. Developing an 'interactive' booklet on respiratory tract infections in children for use in primary care consultations. Patient Educ Couns 2008;73:286-93.

40. Andrews T, Thompson M, Buckley DI, et al. Interventions to influence consulting and antibiotic use for acute respiratory tract infections in children: a systematic review and meta-analysis. PLOS ONE 2012;7:e30334.

41. Thompson Coon J, Martin A, Abdul-Rahman A-K, et al. Interventions to reduce acute paediatric hospital admissions: a systematic review. Arch Dis Child 2012;97:304-11.

42. Nauert R. Stress Affects Learning and Memory. Psychology Central. 2008. (Retrieved on 31 January 2012). http://psychcentral.com/ news/2008/03/12/stress-affects-learning-and-memory/2031.html

43. Action for Sick Children. First contact care survey. London: Action for Sick Children, 2013.

44. Jones C, Neill S, Lakhanpaul M, et al. Information needs of parents for acute childhood illness: determining 'what, how, where and when' of safety netting using qualitative exploration with parents and clinicians. BMJ Open 2014;4:e003874.

45. Neill SJ, Jones CH, Lakhanpaul M, et al. the ASK SNIFF research team. Parent's information seeking in acute childhood illness: what helps and what hinders decision making? Health Expect 2014. Article first published online: 20 Oct 2014 